\title{
Network Bipartivity and the Transportation Efficiency of European Passenger Airlines
}

\author{
Ernesto Estrada ${ }^{1}$ and Jesús Gómez-Gardeñes ${ }^{2}$ \\ 1 Department of Mathematics and Statistics, University of Strathclyde, \\ 26 Richmond Street, Glasgow, G1 1 XH, UK \\ 2 Department of Condensed Matter Physics \& Institute for Biocomputation and Physics of \\ Complex Systems (BIFI) - Universidad de Zaragoza, 50009 Zaragoza, Spain
}

Corresponding author: Ernesto Estrada

Telf. $+44(0) 1415483657$

Fax. $+44(0) 1415483345$

E-mail: ernesto.estrada@strath.ac.uk 


\begin{abstract}
The analysis of the structural organization of the interaction network of a complex system is central to understand its functioning. Here, we focus on the analysis of the bipartivity of graphs. We first introduce a mathematical approach to quantify bipartivity and show its implementation in general and random graphs. Then, we tackle the analysis of the transportation networks of European airlines from the point of view of their bipartivity and observe significant differences between traditional and low cost carriers. Bipartivity shows also that alliances and major mergers of traditional airlines provide a way to reduce bipartivity which, in its turn, is closely related to an increase of the transportation efficiency.
\end{abstract}

Keywords

- Complex Networks

- Bipartivity

- Airline Transportation Systems

- Transportation Efficiency 


\section{Introduction}

A fundamental characteristic of complex systems is that, in general, they are networked. Thus, complex networks, which represent the skeleton of such complex systems, are ubiquitous in many real-world scenarios, ranging from the biomolecular - those representing gene transcription, protein interactions, and metabolic reactions - to the social and infrastructural organization of modern society [1]. Mathematically speaking, these networks are graphs with the nodes representing the entities of the system and the edges representing the "relations" among those entities. From an structuralist point view of nature it could be claimed that a large proportion of the properties of these complex systems are determined by the structure of these networks. The question about what do we mean by "the structure" of these networks is a tricky one. This situation is reminiscent of Edgar Allan Poe response about what is the structure of a strange ship: "What she is not, I can easily perceive-what she is I fear it is impossible to say" [2]. Then, the pragmatic approach used in network theory and beyond is to consider structural invariants which characterize some portions of this structure which in global terms scape to our formal definitions. That is the reason why we have such a large amount of structural invariants, i.e., numbers that characterize some properties of the network independently of the labelling of nodes and edges [3]. Such invariants include the average path length, clustering coefficients, densities, assortativity coefficients, and many more (see $[1,3]$ for non-exhaustive lists).

The concept of network bipartivity is one that have given rise to some structural invariants to characterize how much bipartitivity a network has. Bipartivity has long been studied in graph theory as a black-and-white concept. That is, just by considering that a graph is or is not bipartite. However, in the noughties there were three papers that attempted to characterize how much bipartivity a non-bipartite graph has. The pioneering work of Holme et al. proposed the first of such measures in 2003 by using computational methods [4]. In 2005 Estrada and Rodríguez-Velázquez applied spectral graph theory to develop a mathematical characterization of graph bipartivity [5]. The third work, published by Pisanski and Randić, uses a characterization of network cyclicity to account for bipartivity [6]. In particular the bipartivity index developed by Estrada and Rodríguez-Velázquez has found a different number of applications to network problems ranging from the analysis of fullerene stability to the structure of food webs (see for instance $[7,8,9,10]$. Although this index is mathematically appealing - it is based on well defined matrix functions of the adjacency matrix of a graph - it has some drawbacks. The most important one is that it is bounded between 0.5 and 1.0, which means that it has a very narrow range of values for the analysis of networks. Also importantly, it is based on two different kinds of matrix functions - the exponential and the hyperbolic cosine-, which make its calculation computationally complicated from the point of view os using numerical methods for their calculations.

Here we propose a new mathematical approach to quantify the bipartivity of a network by considering a single matrix function-the exponential. This 
new index is ranged between 0 and 1 . We show a few mathematical properties of this index for general networks as well as for random graphs. We then embarked in the study of the airline transportation networks in Europe to see how the different degrees of bipartivity affect their global efficiency in terms of the number of passengers transported and the number of hours flown. We show that the new bipartivity index accounts very well for the main characteristics of these European airline networks and allow us to understand the main structural differences between traditional and low-cost carriers operating in Europe.

\section{Graph Theoretical Preliminaries}

Here we present some definitions, notations, and properties which will be used in this work (see [1,3]). A graph $G=(V, E)$ is defined by a set of $n$ nodes (vertices) $V$ and a set of $m$ edges $E=\{(u, v) \mid u, v \in V\}$ between the nodes. An edge is said to be incident to a vertex $u$ if there exists a node $v \neq u$ such that either $(u, v) \in E$ or $(v, u) \in E$. The graph is said to be undirected if the edges are formed by unordered pairs of vertices. A walk of length $k$ in $G$ is a set of nodes $i_{1}, i_{2}, \ldots, i_{k}, i_{k+1}$ such that for all $1 \leq l \leq k,\left(i_{l}, i_{l+1}\right) \in E$. A closed walk is a walk for which $i_{1}=i_{k+1}$. A path is a walk with no repeated nodes. A graph is connected if there is a path connecting every pair of nodes. A graph with unweighted edges, no self-loops (edges from a node to itself), and no multiple edges is said to be simple. Throughout this work, we will always consider undirected, simple, and connected networks. In this setting the matrix $A=\left(a_{u v}\right)$, called the adjacency matrix of the graph, has entries

$$
a_{u v}=\left\{\begin{array}{ll}
1 & \text { if }(u, v) \in E \\
0 & \text { otherwise }
\end{array} \quad \forall u, v \in V\right.
$$

and, in the particular case of an undirected network as the ones studied here, the adjacency matrix of the graph is symmetric, $a_{u v}=a_{v u}$, and thus its eigenvalues are real. In the following we label the eigenvalues of $A$ in non-increasing order: $\lambda_{1} \geq \lambda_{2} \geq \ldots \geq \lambda_{n}$. The degree of a node $k_{i}$ is the number of edges incident to that node. Since $A$ is a real-valued, symmetric matrix, we can decompose $A$ into $A=Q \Lambda Q^{T}$ where $\Lambda$ is a diagonal matrix containing the eigenvalues of $A$ and $Q=\left[\mathbf{q}_{1}, \ldots, \mathbf{q}_{n}\right]$ is orthogonal, where $\mathbf{q}_{i}$ is an eigenvector associated with $\lambda_{i}$.

The network density is given by:

$$
\delta=\frac{2 m}{n(n-1)},
$$

where $m$ is the number of edges. Here we will call, as usual in network theory, average path length the average of the shortest path distance in the graph:

$$
\bar{l}=\frac{2}{n(n-1)} \sum_{u \neq v} d(u, v),
$$


where $d(u, v)$ is the shortest path distance between the nodes $u$ and $v$.

An important quantity for the current work is one defined for studying communication processes in networks, which is called communicability function [11]. In particular, the self-communicability function, also known as the subgraph centrality [12] of the corresponding node, is defined as follows. Let $u$ be a node of $G$, then

$$
G_{u u}=\sum_{k=0}^{\infty} \frac{\left(A^{k}\right)_{u u}}{k !}=(\exp (A))_{u u}=\sum_{k=1}^{n} e^{\lambda_{k}} \mathbf{q}_{k}^{2}(u) .
$$

The sum of all subgraph centralities in a network is nowadays known as the Estrada index of a graph [13,14, 15, 16], which is defined as

$$
\operatorname{EE}(G)=\sum_{u=1}^{n}(\exp (A))_{u u}=\operatorname{tr}(\exp (A)) .
$$

By the properties of the matrix exponential and of the trace of a matrix we can easily see that

$$
\mathrm{EE}(G)=\operatorname{tr}(\sinh (A))+\operatorname{tr}(\cosh (A)) .
$$

These functions count the total number of closed walks starting (and ending) at node $u$, weighted in decreasing order of their length $k$ by a factor $\frac{1}{k !}$; therefore it is considering shorter closed walks more influential than longer ones (see $[11,17,18])$. In particular, the hyperbolic sine function counts the number of closed walks of odd length in the graph and the hyperbolic cosine one counts the even-length ones.

\section{Spectral Bipartivity Index in Graphs}

There are a few characterizations of bipartite graphs in graph theory. For instance, the following characterization is a well-known one [19].

Lemma 1. A graph is bipartite if and only if it does not contain any odd cycle.

We now provide a related characterization of bipartite graphs which will be of great usefulness in this work.

Theorem 2. A graph is bipartite if and only if $\operatorname{tr} \sinh (A)=0$.

Proof. Let us consider the Taylor series expansion of $\operatorname{tr} \sinh (A)$

$$
\operatorname{tr} \sinh (A)=\operatorname{tr} A+\frac{\operatorname{tr} A^{3}}{3 !}+\cdots=\sum_{k=0}^{\infty} \frac{\operatorname{tr}\left(A^{2 k+1}\right)}{(2 k+1) !} .
$$

We know that $\operatorname{tr}\left(A^{2 k+1}\right)$ counts the number of closed walks of length $2 k+1$ in the graph. Every closed walk of odd length necessarily involves an odd cycle. Then, because in a bipartite graph there are no odd cycles, $\operatorname{tr}\left(A^{2 k+1}\right)=0$ for all $k$, which proves the above result. 
Let us call frustrated closed walk to a closed walk which involves any odd cycle in the network. Similarly, a non-frustrated closed walk is the one which

does not involve any odd cycle. Let us now consider a normalized measure of the difference between the number of non-frustrated and frustrated walks:

$$
b_{s}=\frac{W^{N}-W^{F}}{W^{N}+W^{F}} .
$$

The use of the term frustrated to designate closed walks involving any odd cycle in the network comes from its use in spin systems. In those cases there are $n$ magnetic ions residing on the corners of a cycle with antiferromagnetic interactions between them; the energy is minimized when each spin is aligned opposite to neighbors. In those systems with odd cycles once the first $n-1$ spins align anti-parallel, the $n$th one is frustrated because its two possible orientations, up and down, give the same energy (see for instance [20]).

Using the properties of the Estrada index that we have stated in the previous section we can find a natural way of accounting for this normalized measure. This is carried out by using the traces of the hyperbolic cosine and sine matrix functions. That is, we propose to consider

$$
b_{s}=\frac{\operatorname{tr} \cosh (A)-\operatorname{tr} \sinh (A)}{\operatorname{tr} \cosh (A)+\operatorname{tr} \sinh (A)},
$$

that, by considering that $A$ is diagonalizable, can be re-written as:

$$
b_{s}=\frac{t r \exp (-A)}{t r \exp (A)}=\frac{\sum_{j=1}^{n} \exp \left(-\lambda_{j}\right)}{\sum_{j=1}^{n} \exp \left(\lambda_{j}\right)} .
$$

Notice, that the numerator is the Estrada index for the negative of the adjacency matrix and the denominator is the 'classical' Estrada index of a graph. Hereafter, we call $b_{s}$ the spectral bipartivity index of the graph.

\section{Mathematical Properties of the Spectral Bipartivity Index}

We now analyze the mathematical properties of the introduced spectral bipartivity index $b_{s}$.

Proposition 3. The spectral bipartivity index is bounded as

$$
0<b_{s} \leq 1,
$$

where the upper bound is reached for any bipartite network and the lower bound is reached asymptotically for a complete graph with $n \rightarrow \infty$.

Proof. It is easy to show that the upper bound is reached for any bipartite graph for which $\operatorname{tr} \sinh (A)=0$. For the upper bound let us consider the complete graph $K_{n}$. Then,

$$
b_{s}\left(K_{n}\right)=\frac{\exp (-(n-1))+(n-1) \exp (-1)}{\exp (n-1)+(n-1) \exp (1)},
$$


so that we have that:

$$
\lim _{n \rightarrow \infty} b_{s}\left(K_{n}\right)=0
$$

Let $G=(V, E)$ be a graph having $n$ nodes. Let us assume that $G \neq K_{n}$, i.e., $G$ is not a complete graph. The best bipartition of $G$ consists in splitting the set of nodes $V$ into two non-empty sets $V_{1}$ and $V_{2}\left(V=V_{1} \cup V_{2}\right)$, such as the number of edges $e=(i, j)$ in which $i \in V_{k}$ implies that $j \in V_{k}, k=\{1,2\}$ is minimized. Let

$$
\alpha(G)=\alpha=\frac{\sum_{j=1}^{n} \sinh \left(\lambda_{j}\right)}{\sum_{j=1}^{n} \cosh \left(\lambda_{j}\right)},
$$

be the ratio of odd to even closed walks in $G$. Then, we have the following result.

Proposition 4. Let $G$ be a graph with best bipartition given by the sets $V_{1}$ and $V_{2}$. Let $G+e$ be the graph resulting from adding the edge e to $G$, such that a and $b$ are the contributions of the edge e to even and odd closed walks in $G+e$ , respectively. Then, if $b \geq \alpha a$

$$
b_{s}(G) \geq b_{s}(G+e) .
$$

Proof. The spectral bipartivity index of $G+e$ is

$$
\begin{aligned}
b_{s}(G+e) & =\frac{\left(\sum_{j=1}^{n} \cosh \left(\lambda_{j}\right)+a\right)-\left(\sum_{j=1}^{n} \sinh \left(\lambda_{j}\right)+b\right)}{\sum_{j=1}^{n} \exp \left(\lambda_{j}\right)+a+b} . \\
& =\frac{\sum_{j=1}^{n} \cosh \left(\lambda_{j}\right)+a}{\sum_{j=1}^{n} \exp \left(\lambda_{j}\right)+a+b}-\frac{\sum_{j=1}^{n} \sinh \left(\lambda_{j}\right)+b}{\sum_{j=1}^{n} \exp \left(\lambda_{j}\right)+a+b}
\end{aligned}
$$

Since $\cosh x>\sinh x, \forall x$,

$$
\sum_{j=1}^{n} \cosh \left(\lambda_{j}\right)>\sum_{j=1}^{n} \sinh \left(\lambda_{j}\right)
$$

and because $b \geq \alpha a$, we have that

$$
b \sum_{j=1}^{n} \cosh \left(\lambda_{j}\right) \geq a \sum_{j=1}^{n} \sinh \left(\lambda_{j}\right) .
$$

Adding $\left(b+\sum_{j=1}^{n} \exp \left(\lambda_{j}\right)\right)\left(\sum_{j=1}^{n} \sinh \left(\lambda_{j}\right)\right)$ to both sides of the previous inequality, we get

$$
\left(\sum_{j=1}^{n} \exp \left(\lambda_{j}\right)\right)\left(b+\sum_{j=1}^{n} \sinh \left(\lambda_{j}\right)\right) \geq\left(\sum_{j=1}^{n} \sinh \left(\lambda_{j}\right)\right)\left(a+b+\sum_{j=1}^{n} \exp \left(\lambda_{j}\right)\right),
$$


which means that

$$
\frac{b+\sum_{j=1}^{n} \sinh \left(\lambda_{j}\right)}{a+b+\sum_{j=1}^{n} \exp \left(\lambda_{j}\right)} \geq \frac{\sum_{j=1}^{n} \sinh \left(\lambda_{j}\right)}{\sum_{j=1}^{n} \exp \left(\lambda_{j}\right)} .
$$

In a similar way we can prove that

$$
\frac{\sum_{j=1}^{n} \cosh \left(\lambda_{j}\right)}{\sum_{j=1}^{n} \exp \left(\lambda_{j}\right)} \geq \frac{a+\sum_{j=1}^{n} \cosh \left(\lambda_{j}\right)}{a+b+\sum_{j=1}^{n} \exp \left(\lambda_{j}\right)} .
$$

Combining the inequalities 6 and 7 we finally obtain:

$$
\begin{aligned}
b_{s}(G) & =\frac{\sum_{j=1}^{n} \cosh \left(\lambda_{j}\right)}{\sum_{j=1}^{n} \exp \left(\lambda_{j}\right)}-\frac{\sum_{j=1}^{n} \sinh \left(\lambda_{j}\right)}{\sum_{j=1}^{n} \exp \left(\lambda_{j}\right)} \\
\geq & \frac{a+\sum_{j=1}^{n} \cosh \left(\lambda_{j}\right)}{a+b+\sum_{j=1}^{n} \exp \left(\lambda_{j}\right)}-\frac{b+\sum_{j=1}^{n} \sinh \left(\lambda_{j}\right)}{a+b+\sum_{j=1}^{n} \exp \left(\lambda_{j}\right)}=b_{s}(G+e)
\end{aligned}
$$

Remark 5. The condition $b \geq \alpha a$ indicates that the edge added to $G$ increases more the contribution to odd than to even closed walks relative to the ratio of these walks in the original graph $G$. This is always the case when the edge $e$ is added between pairs of nodes which are both in the same partition $V_{1}$ or $V_{2}$ of $G$, that is, when the edge $e=(i, j)$ is such that $i \in V_{k}$ implies that $j \in V_{k}, k=\{1,2\}$. If the edge $e=(i, j)$ is added to connect two nodes in distinct sets, i.e., $i \in V_{1}, j \in V_{2}$, then the condition condition $b \geq \alpha a$ is not necessarily fulfilled. In this case it is possible that the bipartivity of the network can increase.

The Proposition 4 proves that the spectral bipartivity index decays monotonically when the number of frustrated edges increases in the network. In Figure 1 we illustrate this for a toy graph of $n=5$ nodes.

We now move to the analysis of bipartivity in Erdôs-Rényi (ER) random graphs. In this case we prove the following result.

Theorem 6. Let $G(n, p)$ be an Erdös-Rényi random graph with $n$ nodes and probability $p$. When $n \rightarrow \infty$, the bipartivity of $G(n, p)$ is given by

$$
b_{s}(E R)= \begin{cases}1 & \text { if }(n p) \rightarrow 0 \\ 0 & \text { if }(n p) \rightarrow \infty .\end{cases}
$$

Proof. Let $\lambda_{1}>\lambda_{2} \geq \cdots \geq \lambda_{n}$ be the eigenvalues of the adjacency matrix of the ER graph. When $n \rightarrow \infty: \lambda_{1}=n p$ and $\lambda_{j \geq 2}=0$. Thus,

$$
b_{s}(E R)=\frac{n-1+\exp (-n p)}{n-1+\exp (n p)}=\frac{n-1}{n-1+\exp (n p)}+\frac{\exp (-n p)}{n-1+\exp (n p)} .
$$

By applying the limit when $n \rightarrow \infty$ the two dominating regimes are observed, which proves the result. 


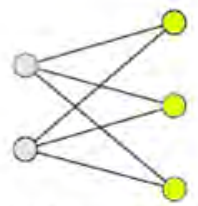

(a) $b_{s}=1.000$

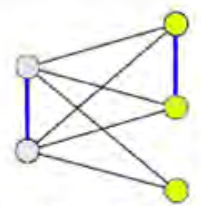

(e) $b_{s}=0.383$

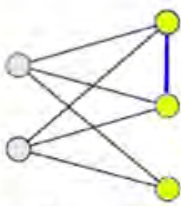

(b) $b_{s}=0.658$

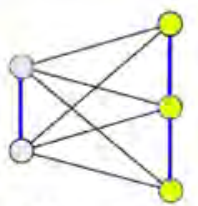

(f) $b_{s}=0.289$

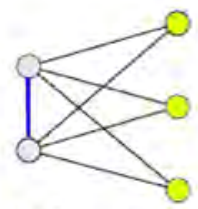

(c) $b_{s}=0.538$

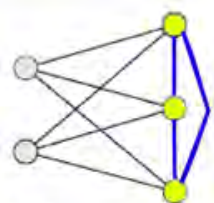

(g) $b_{s}=0.289$

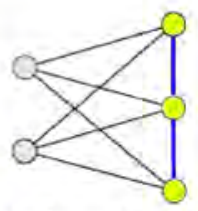

(d) $b_{s}=0.462$

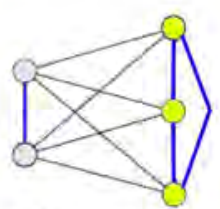

(h) $b_{s}=0.194$

Figure 1: Illustration of the monotonic decay of the spectral bipartivity index in a graph of $n=5$ nodes. In (a) we show a perfectly bipartite graph whereas from (b) to (h) the number of frustrated edges is progressively increased. As show the addition of frustrated edges leads to the increase of the spectral bipartivity index $b_{s}$

The previous result indicates that an ER graph is almost bipartite, i.e., $b_{s}(E R) \simeq 1$ if

$$
p \ll \frac{\ln (n-1)}{n} .
$$

We should consider that an ER random graph with such small density could be disconnected. It is well known that if $p=(\log n+c) / n$, where $c$ is an arbitrary constant, then $\operatorname{Pr}[G(n . p)$ is connected $] \rightarrow \exp (-\exp (-c))$ as $n \rightarrow \infty$. However, it is possible to obtain connected ER random graphs with relatively large bipartivity by working in a very low-density regime (we recall that in an ER graph $p=\delta$, where $\delta$ is the graph density). In closing, the previous result indicates that there is a transition between a bipartite graph to a highly nonbipartite one in the ER model, which is controlled by the graph density. That is, for fixed $n$, an ER graph is close to bipartite when the density of the graph is very small. As soon as this density approaches 1, the graph becomes highly non-bipartite as a consequence that it is approaching the complete graph.

In the Fig. (2) we illustrate the plot of the spectral bipartivity index versus the graph density $\delta$ for ER random graphs with 500, 1000 and 2000 nodes. Every point is the result of averaging 100 random realizations of the ER graph with the same number of nodes and edges. As can be seen the spectral bipartivity index decays very fast with the increase of the graph density. Such decay is faster as the number of nodes increases as predicted by our previous analytic result. Thus, highly bipartite random ER graphs are only possible in a very low density regime, which is realizable when the number of nodes is very large. 


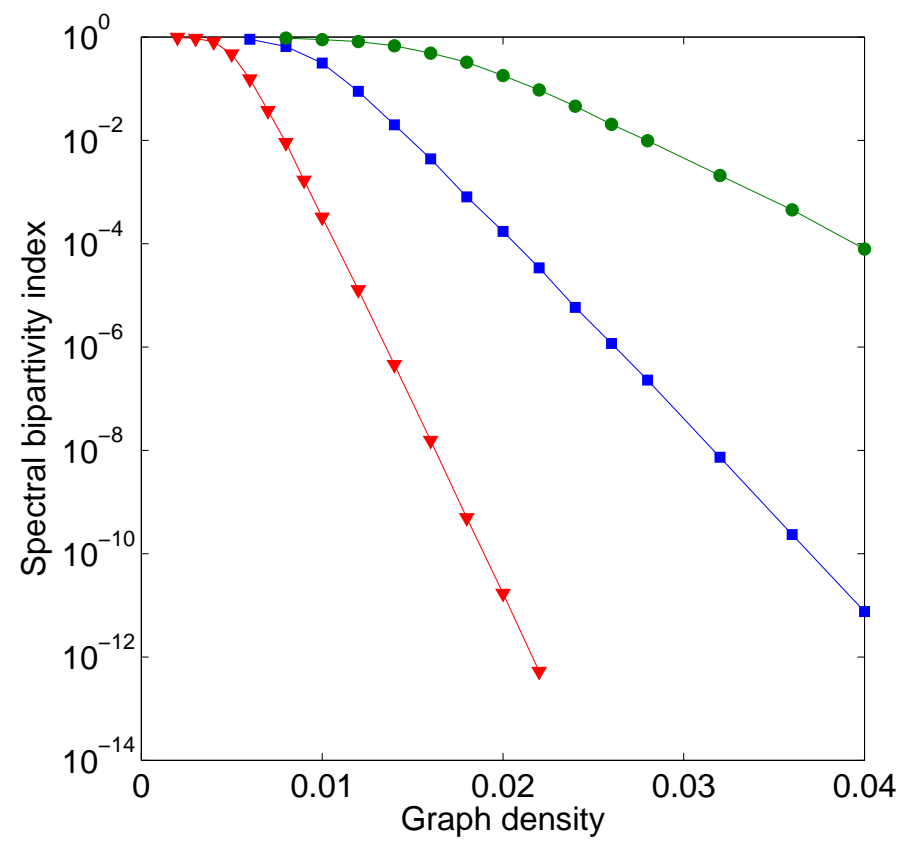

Figure 2: Scatterplot of the spectral bipartivity index versus the graph density for ER random graphs with $n=500$ (green circles), $n=1000$ (blue squares), and $n=2000$ (red triangles). The lines joining the points are used to guide the eye. The plot is in semilogarithmic scale. 


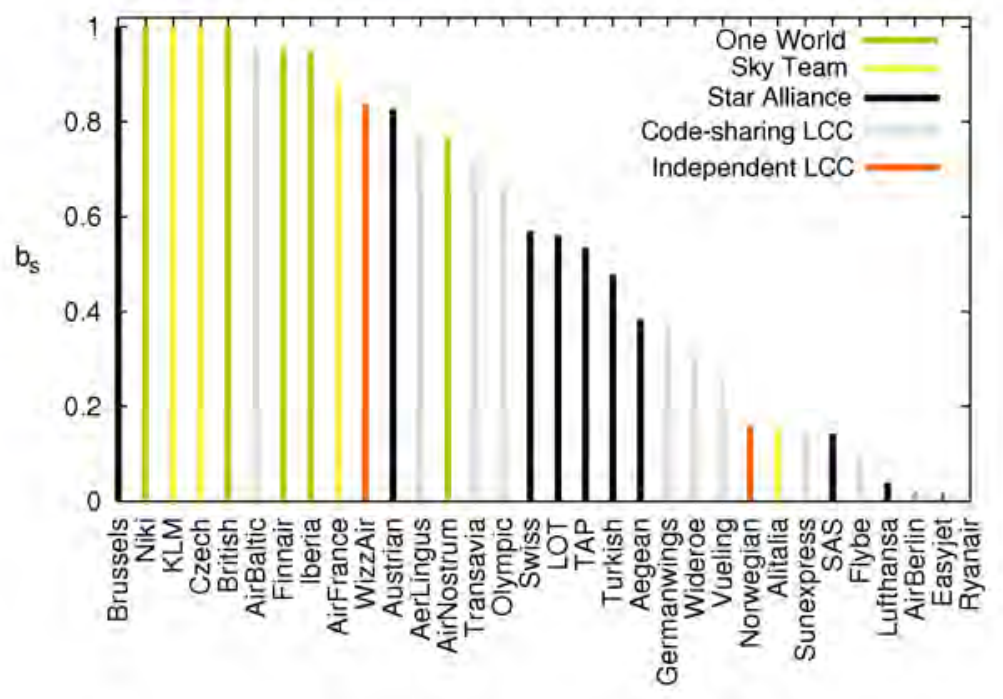

Figure 3: Ranking of the airlines ordered (from larger to smaller) by their bipartivity $b_{s}$. Note that traditional airlines are those with the largest bipartivity values.

\section{Analysis of European Passenger Airlines}

Armed with the previous method to quantify bipartivity in a network we tackle the problem of evaluating this property in real networks. To this aim we consider the European air transportation system [21, 22] represented by 33 passenger airlines, 25 of which correspond to 'traditional' and 8 to 'low-cost' carriers (LCCs). For each airline carrier we consider a network in which the nodes represent any of the 450 commercial airports existing in Europe and two nodes (airports) are connected if the corresponding airline has a flight between them. A resume of the main characteristics of these airline networks are displayed in the Table 1.

Our goal is to show, by means of the spectral bipartivity index, the organizational differences of these two types of airlines and how the alliances between traditional airilnes affect the value of bipartivity. First, in Fig. 3 we show the bipartivity ranking of the set of 33 airlines. Remarkably, traditional airlines display high bipartivity indexes, reaching $b_{s} \simeq 1$ in the cases of Brussels, Niki, KLM, Czech and British. On the orher hand, most of LCCs display small values being almost zero for the most important ones: Easyjet and Ryanair.

The presence of low-cost carriers (LCC) in Europe represents a significant change in the evolution of airline industry. Although most traditional carriers have reported heavy losses and capacity reduction in recent years, LCCs have been continuously expanding in the European short-haul market. The average number of airports $\langle n\rangle$ and flights per airline $\langle m\rangle$ are given in Table 2 for both traditional and LCCs. Both types of networks (traditional and LC) have 


\begin{tabular}{|c|c|c|c|c|}
\hline Airline & $n$ & $m$ & $\delta$ & $\bar{l}$ \\
\hline Easyjets & 99 & 307 & 0.063 & 2.345 \\
\hline Flybe & 43 & 99 & 0.110 & 2.277 \\
\hline GermanWings & 42 & 66 & 0.077 & 2.049 \\
\hline Norwegian & 52 & 87 & 0.066 & 2.207 \\
\hline Ryanair & 128 & 601 & 0.074 & 2.247 \\
\hline Transavia & 40 & 57 & 0.073 & 2.428 \\
\hline Vueling & 36 & 63 & 0.100 & 2.186 \\
\hline WizzAir & 45 & 92 & 0.092 & 2.663 \\
\hline Aegean & 38 & 53 & 0.075 & 2.195 \\
\hline AirBaltic & 45 & 45 & 0.045 & 2.082 \\
\hline AirBerlin & 75 & 184 & 0.066 & 2.374 \\
\hline AirFrance & 59 & 69 & 0.043 & 2.194 \\
\hline Airlingus & 45 & 58 & 0.059 & 2.108 \\
\hline AirNostrum & 48 & 69 & 0.061 & 2.512 \\
\hline Alitalia & 51 & 93 & 0.073 & 1.972 \\
\hline Austrian & 67 & 72 & 0.033 & 2.022 \\
\hline British & 63 & 65 & 0.033 & 2.759 \\
\hline Brussels & 44 & 43 & 0.046 & 1.954 \\
\hline Czech & 42 & 41 & 0.048 & 1.952 \\
\hline FinnAir & 42 & 42 & 0.049 & 1.996 \\
\hline Iberia & 35 & 35 & 0.059 & 1.995 \\
\hline KLM & 63 & 62 & 0.032 & 1.968 \\
\hline Lufthansa & 106 & 244 & 0.044 & 2.163 \\
\hline Malev & 35 & 34 & 0.057 & 1.943 \\
\hline NIKI & 36 & 37 & 0.059 & 2.441 \\
\hline Olympic & 37 & 43 & 0.065 & 1.985 \\
\hline Panagra & 45 & 58 & 0.059 & 2.194 \\
\hline Polish & 44 & 55 & 0.058 & 2.026 \\
\hline Scandinavian & 66 & 110 & 0.051 & 2.312 \\
\hline Swiss & 48 & 60 & 0.053 & 1.947 \\
\hline TAP & 42 & 53 & 0.062 & 1.971 \\
\hline Turkish & 86 & 118 & 0.032 & 2.016 \\
\hline Windroe & 45 & 90 & 0.091 & 3.345 \\
\hline
\end{tabular}

Table 1: Some topological invariants for the 33 airline carrier networks studied in this work. We give the number of airport $n$ used by each company, number of flights $m$, network density $\delta$, average path length $\bar{l}$ and global clustering coefficient $C$. 
very similar number of nodes, densities and average path length (see Table 1). However, the values obtained for the average bipartite index point out that LCCs have networks which are $34 \%$ less bipartite than those used by traditional airlines.

\subsection{Multilayer Approach}

Another characteristic of European airlines is that many of them are grouped into alliances with the goal of expanding their networks and to reduce costs. These alliances include One World, Sky Team and Star Alliance. In Fig. 3 we have indicated (see colors) the alliance of each of the traditional airlines. It is interesting that those airlines belonging to Sky Team and One World groups show large values of bipartivity whereas most of the airlines forming the Star Alliance display smaller values, lying in the range where most LCCs are.

From the network perspective, alliances are multiplex networks [23, 24] so that each airline constitutes an interaction layer and each airport is a node that is present in each of the layers. A common way to study how the different layers of a multiplex interplay is to aggregate them into a single network comprising the same set of nodes and the different links that connect them. Thus, here we compute the bipartivity index of an alliance as the bipartivity of the graph resulting after aggregating all the layers corresponding to the airlines that participate of it.

In Table 3 we show the bipartivity of the three alliances (computed by merging all the layers into a single one, the mergin is carried out in an unweighted way, i.e., a one is placed in the adjacency matrix if there is a link in any layer, zero otherwise) and the average bipartivity of the airlines (layers) composing each of them. As can be seen the bipartivity drops significatly when merging the airlines within an alliance, specially in the case of Star Alliance, which in its turn contains the airlines with smaller average bipartivity. Thus, in spite of the large bipartivity values displayed by traditional airlines, the formation of alliances can be seen as a form of increasing the frustration level of their networks, i.e., decreasing their network bipartivity.

The merging of all the layers (airlines) composing a multiplex (alliance) is a very strong assumption that considers that all the carriers in one alliance use simultaneously all the networks provided by the rest of the carriers in that alliance. On the contrary, the fact that two airlines belong to the same alliance does not imply that all the flights operated by them are under a codeshare agreement. However, in the recent years there have been a couple of major

\begin{tabular}{ccccc}
\hline & $\langle n\rangle$ & $\langle m\rangle$ & $\langle\delta\rangle$ & $\left\langle b_{s}\right\rangle$ \\
\hline \hline Low-cost & 61 & 172 & 0.081 & 0.305 \\
\hline Traditional & 52 & 74 & 0.055 & 0.643 \\
\hline
\end{tabular}

Table 2: Main descriptors of the graphs associated to LCCs and traditional carriers operating in the the European Air Transportation System. 


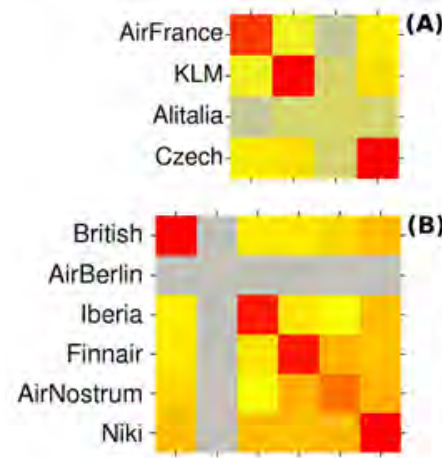

(A)

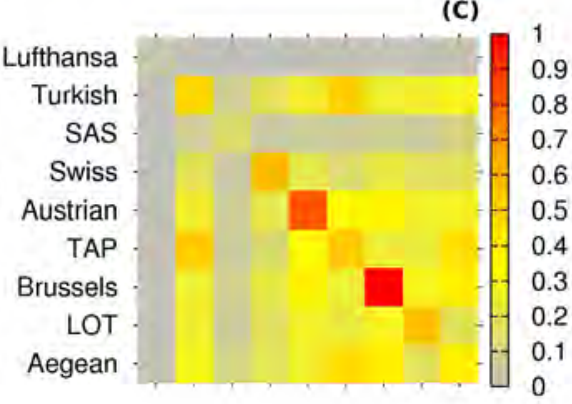

Figure 4: Bipartibity of all the possible two-airline combinations within each of the alliances of traditional carriers. (A) Sky Team, (B) One World and (c) Star Alliance.

mergers in the European airline market which, from the network perspective, fulfill the assumptions behind the aggregation of layers.

To analyze what are the effects that the merging of couples of traditional airlines has on their bipartivity we have analyzed all the possible 2-airlines coalitions within an Alliance. In Fig. 4 we show the corresponding bipartivity indexes in three color-coded matrices (one per alliance) so that the element $(i, j)$ of these matrices reveals the value of the bipartivity index when aggregating airline $i$ and airline $j$ into a single network. In Fig. 4.A we plot the bipartivity matrix corresponding to the Sky Team alliance. It is remarkable that Air France and KLM show very high bipartivity indexes when isolated (0.8773 and 1.000 respectively) whereas the single merging of them yields a bipartivity index of 0.2799. The other major merger between Iberia and British Airways is reported in Fig. 4.B (One World alliance) which isolated display large bipartivities (0.9461 for Iberia and 1.000 for British Airways) while merged reach a value of 0.3868.

It is remarkable, that for both Sky Team and One World alliances, the two mergers reported above are those yielding the smaller bipartivity value among those created from the coalition of large bipartivity airlines (Air France, KLM and Czech for Sky Team and British, Iberia, Finnair and Nikki for One World).

In the case of Star Alliance, Fig. 4.C, the diagonal reveal that most of the

\begin{tabular}{|c|c|c|c|c|}
\hline & $m$ & $n$ & $b_{s}$ & $\left\langle b_{s}\right\rangle$ \\
\hline \hline One World & 6 & 148 & 0.001 & 0.78 \\
\hline Star Alliance & 9 & 212 & $6 \cdot 10^{-6}$ & 0.50 \\
\hline Sky Team & 4 & 118 & 0.022 & 0.76 \\
\hline
\end{tabular}

Table 3: Main descriptors of the graphs associated to LCCs and traditional carriers operating in the the European Air Transportation System. The values of $b_{s}$ correspond to the bipartivity of the merged multiplexes and $\left\langle b_{s}\right\rangle$ to the average bipartivity of all the individual airlines in a given alliance. 


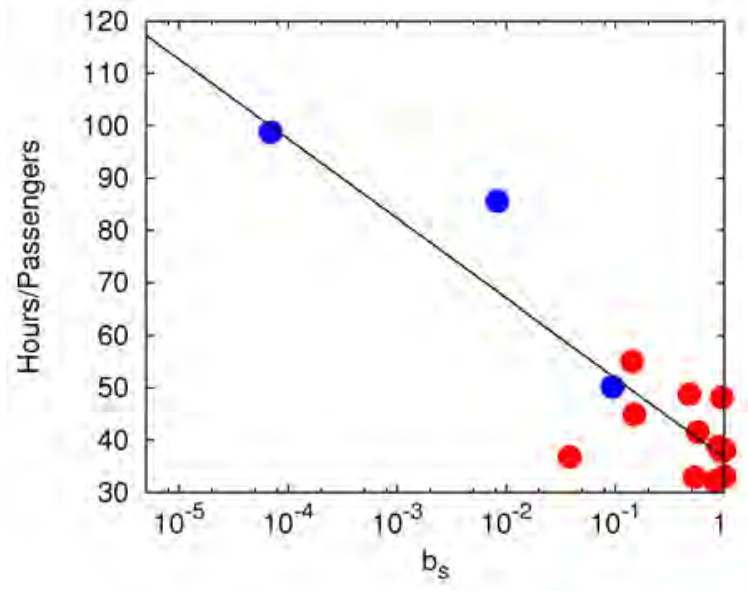

Figure 5: Illustration of the relation between the airline carriers efficiency and the spectral bipartivity index. The airline efficiency is measured by the number of passengers transported divided by the number of hours flown. Blue nodes correspond to low-cost companies and red nodes to traditional ones.

airlines contained in it show moderate bipartivity values, at variance with One World and Sky Team. This result was already reported in Fig. 3 and Table 3. Remarkably, only two airlines (Austrian and Brussels) show very high bipartivity indexes ( 0.8256 and 1.000 respectively) so that one would be tempted to say that a merger between these two airlines, yielding a bipartivity of 0.3717 , would be beneficial for both airlines and the alliance itself.

\subsection{Bipartivity versus Transportation Efficiency}

It is easily imaginable that there are many economic drivers for the merger of two airline companies. However, it is reasonable to think, on the basis of the previous results, that increasing the level of frustration of the merged network plays a fundamental role in increasing the productivity of the newly formed group. For instance, let us consider a passenger who plans to visit Paris and Amsterdam departing from Bucharest. By using Air France she can travel directly from Bucharest to Paris and then to Amsterdam. At this point she has to return to Paris to take a returning flight to Bucharest and totaling $4,370 \mathrm{~km}$ $(2,716 \mathrm{mi})$. However, using a shared code with Air France-KLM she can flight Bucharest-Paris-Amsterdam with Air France and return directly from Schiphol airport to Bucharest using KLM. This route totals 4,038 km (2,509 mi), which is $332 \mathrm{~km}$ (207 mi) shorter than the previous one. Thus, the merging of the two companies in this case has decreased the bipartivity of this route by creating a triangle that connects the three locations using a combination of both carriers.

Consequently, we can measure the transportation efficiency, Eff as the ratio of the number of passengers to the number of hours flown by a carrier. Then, we can see that decreasing the bipartivity of a network should increase the efficiency 
by transporting the same number of passengers by using less number of hours flown. In fact, this is exactly what we observe when the number of passengers (in millions) divided by hours flown is plotted versus the bipartivity of the air transportation networks of 15 traditional and LCCs in Europe (see Figure 5). The linear fit is given by the equation:

$$
\mathrm{Eff} \approx-6.61 \cdot \log b_{s}+36.59
$$

with Pearson correlation coefficient -0.888 . This correlation points out to the fact that the lack of bipartivity in the transportation network is a major structural driver for the efficiency of the passenger airline carriers studied here.

\section{Conclusions}

We have proposed a novel way for calculating the bipartivity of a network that relies on a single function of the adjacency matrix. This approach allows an efficient and fast calculation of bipartivity that can be easily generalized to more complex interaction frameworks. After showing the mathematical properties of bipartivity in general and random graphs we have tackled the problem of analyzing the bipartivity of a set comprising 33 real air transportation networks operating in Europe. By computing the bipartivity of each airline we have observed significant differences between traditional carriers and the low cost airlines, being the bipartivity of the latter ones much smaller than that of the former. Then, we have shown that alliances and major mergers of traditional airlines lead to a decrease of bipartivity.

Motivated by the above findings we have observed that traffic efficiency (as revealed from real data about the traffic flow of each airline) is strongly and negatively correlated with the bipartivity of its network. Therefore, bipartivity provides a good descriptor of the efficiency of transportation networks and can be used to test the goodness of alliances and possible mergers of airlines. The general way of computing bipartivity may allow its use to evaluate more complex mixing of transportaion networks such as the sharecodes of flights between different airlines, which is the usual collaboration benchmark in air transportation systems.

\section{Acknowledgements}

EE thanks the Royal Society of London for a Wolfson Research Merir Award. JGG acknowledges financial support from the European Commission through FET IP projects MULTIPLEX (Grant No. 317532) and PLEXMATH (Grant No. 317614), the Spanish MINECO through the Ramón y Cajal program and Projects FIS2011-25167 and FIS2012-38266, the Comunidad de Aragón (FENOL group), and the Brazilian CNPq through the grant PVE of the Ciencias Sem Fronteiras program.

[1] E. Estrada, The Structure of Complex Networks. Theory and Applications. (Oxford University Press, Oxford, 2011). 
[2] E. A. Poe, Complete Tales and Poems. (Race Point Publishing, 2014).

[3] L.F. Costa, O.N. Oliveira Jr, G. Travieso, F.A. Rodrigues, P.R. Villas Boas, L. Antiqueira, M. P. Viana, and L. E. Correa Rocha, Analyzing and modeling real-world phenomena with complex networks: a survey of applications, Adv. Phys. 60 (2011) 329-412.

[4] P. Holme, F. Liljeros, C. R. Edling, B. J. Kim, Network bipartivity. Phys. Rev. E 68 (2003) 056107.

[5] E. Estrada, J. A. Rodríguez-Velázquez, Spectral measures of bipartivity in complex networks, Phys. Rev. E 72 (2005) 046105.

[6] T. Pisanski, M. Randić, Use of the Szeged index and the revised Szeged index for measuring network bipartivity, Discr. Appl. Math. 158 (2010) 1936-1944.

[7] E. Estrada, Protein bipartivity and essentiality in the yeast protein-protein interaction network, J. Proteome Res. 5 (2006) 2177-2184.

[8] D. M. Walker, A. Tordesillas, Topological evolution in dense granular materials: a complex networks perspective. Int. J. Solids Struct. 47 (2010) 624-639.

[9] T. Doslić, Bipartivity of fullerene graphs and fullerene stability, Chem. Phys. Lett. 412 (2005) 336-340.

[10] E. Estrada, Food webs robustness to biodiversity loss: the roles of connectance, expansibility and degree distribution. J. Theor. Biol. 244 (2007) 296-307.

[11] E. Estrada, and N. Hatano, Communicability in complex networks, Phys. Rev. E 77 (2008) 036111.

[12] E. Estrada, J. A. Rodríguez-Velázquez, Subgraph centrality in complex networks. Phys. Rev. E, 71 (2005) 056103.

[13] E. Estrada, Characterization of 3D molecular structure. Chem. Phys. Lett. 319 (2000) 713-718.

[14] J. A. de la Peña, I. Gutman, J. Rada, Estimating the Estrada index. Lin. Algebra Appl. 427 (2007) 70-76.

[15] H. Deng, S. Radenković, I. Gutman, The Estrada index. Applications of Graph Spectra, Math. Inst., Belgrade, 123-140 (2009).

[16] I. Gutman, H. Deng, S. Radenković, The Estrada index: an updated survey. Selected Topics on Applications of Graph Spectra, Math. Inst., Beograd, 155-174 (2011).. 
[17] E. Estrada, N. Hatano, M. Benzi, The physics of communicability in complex networks. Phys. Rep. 514 (2011) 89-119.

[18] E. Estrada, D. J. Higham, Network properties revealed through matrix functions, SIAM Rev. 52 (2010) 696-714.

[19] R. Diestel, Graph Theory, Grad. Texts in Math. Springer, 2005.

[20] J. F. Sadoc, R. Mosseri, Geometrical Frustration, Cambridge Univ. Press, 1999 ,

[21] A. Cardillo, J. Gómez-Gardeñes, M. Zanin, M. Romance, D. Papo, F. del Pozo, S. Boccaletti, Emergence of network features from multiplexity, Sci. Rep. 3 (2013) 1344.

[22] http://complex.unizar.es/ atnmultiplex/

[23] S. Boccaletti, G. Bianconi, R. Criado, CI. Del Genio, J. Gómez-Gardeñes, M. Romance, I. Sendina-Nadal, Z. Wang, M. Zanin, The structure and dynamics of multilayer networks, Phys. Rep. 544 (2014) 1-122.

[24] M. Kivelä, A. Arenas, M. Barthelemy, J. P. Gleeson, Y. Moreno, M. A. Porter, Multilayer networks, J. Complex Net. 2 (2014) 203-271. 